\title{
Kinematic Analysis and Simulation of a Novel 2T1R Parallel Mechanism
}

\author{
Ding Ding ${ }^{\mathrm{a}}$, Yanbin Zhang ${ }^{\mathrm{*}}$, Xin $\mathrm{Wu}^{\mathrm{c}}$ and Zenghui Wang ${ }^{\mathrm{d}}$
}

School of Mechatronics Engineer, Henan University of Science and Technology, Luoyang, China

azuckerr@hotmail.com, byanbin_zh@163.com (Corresponding Author), ckdwuxin@163.com,

d15236105186@163.com

Corresponding Author: Yanbin Zhang

Keywords: parallel manipulator, kinematic analysis, virtual prototype, simulation

\begin{abstract}
In this research work, a novel spatial parallel manipulator with three degrees of freedom (DOF) is proposed. The moving platform has two-translation and one-rotational DOFs with respect to the fixed base. Based on the position and orientation characteristics (POC) theory, the number of the DOF is computed and the output motion characteristics of the parallel mechanism are analyzed. The formulas for solving position and orientation of the platform are derived. By using the kinematic influence coefficient method, solutions of the forward/inverse velocity and acceleration are derived. The virtual prototype model of the 2T1R parallel manipulator is established by use of Pro/E software. At last, the position, velocity and acceleration curves are described based on ADAMS software.
\end{abstract}

\section{Introduction}

Since the limited-DOF parallel mechanisms (PMs) have some advantages in terms of simple structure, easy control and low costs, it has been become a hot focus in the fields of international mechanisms and robots. Among the limited-DOF PM, the 3-DOF parallel mechanism has been paid more attentions, especially 3-DOF spherical and 3-DOF translation PMs, such as Delta robot [1] and "agile eye" robot [2]. Spatial parallel mechanism with two-translation and one-rotational (2T1R) DOFs, however, has been obtained less attentions in literatures. Hunt [3] proposed the 3-PRS PM in 1983. Refaat and Herve [4] have synthesized of several spatial mechanism; Zhang [5, 6] presented structural synthesis of fully-isotropic 2T1R PM.

In this paper, a novel 3-DOF parallel mechanism with 2T1R DOFs is presented. Based on the POC theory [7], mobility of the mechanism is computed and the output motion characteristics of the platform is analyzed. Then the generalized velocity and the acceleration expressions are set up by using the kinematic influence coefficient method. The virtual prototype model of the 2T1R parallel mechanism is established based on PRO/E software and kinematics simulation is performed by use of ADAMS software.

\section{Structure design}

\subsection{Constructional design.}

The novel 2T1R parallel mechanism proposed here is shown in Fig. 1.The mechanism consists of three chains, a fixed platform and a moving platform. The structure of first chain is $\mathrm{R}_{11} \mathrm{P}_{12} \mathrm{~S}_{13}$ from the base to platform in sequence, second chain is $\mathrm{R}_{21} \mathrm{P}_{22} \mathrm{~S}_{23}$, and the third chain is $\mathrm{C}_{31} \mathrm{P}_{32} \mathrm{R}_{33}$. Here, $\mathrm{R}, \mathrm{P}, \mathrm{S}$ and $\mathrm{C}$ denote revolute joint, prismatic pair, spherical joint and cylindrical pair, respectively. In the first chain, the axis of joint $R_{11}$ is perpendicular to the central line of joint $P_{12}$. In the second, the axis of $R_{21}$ is perpendicular to the central line of $P_{22}$. As for the third chain is concerned, the axis of $C_{31}$ is parallel to the $\mathrm{R}_{33}$ and perpendicular to the central line of $\mathrm{P}_{32}$. In order to achieve the desired output characteristics of the mechanism, three chains should meet the following structural conditions: the axis of the $R_{11}$ is parallel to the axis of the $R_{21}$ and perpendicular to the axis of $C_{31}$. 


\subsection{Degree of freedom of the parallel mechanism}

In this paper, DOF analysis is performed based on the POC theory. Topological structure of the

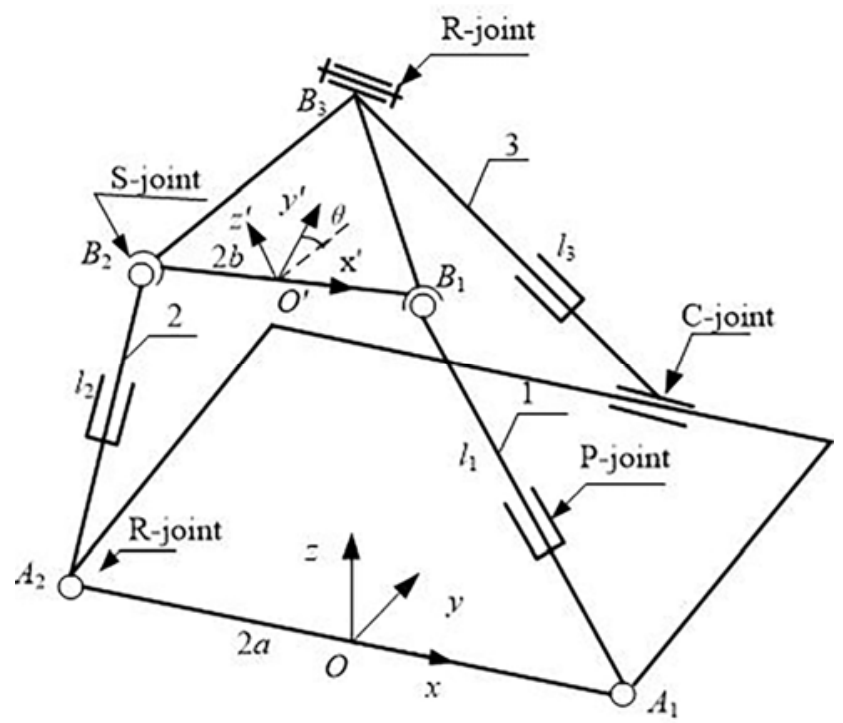

Fig.1 Diagram of the novel 2T1R parallel mechanism

mechanism can be described as follows

the first chain: $\operatorname{soc}\left\{-\mathrm{R}_{11} \perp \mathrm{P}_{12}-\mathrm{S}_{13^{-}}\right\}$,

the second chain: $\operatorname{soc}\left\{-\mathrm{R}_{21} \perp \mathrm{P}_{22}-\mathrm{S}_{23}-\right\}$,

the third chain: $\operatorname{soc}\left\{-\mathrm{C}_{31}\left(\perp \mathrm{P}_{32}\right) / / \mathrm{R}_{33}\right\}$,

where "soc" means single-opened-chain, symbol "//" and " $\perp$ " denote the axes of the neighboring joints being parallel or perpendicular to each other.

Joints $\mathrm{R}_{11}, \mathrm{R}_{21}$ and $\mathrm{C}_{31}$ are mounted on the fixed base. Joints $\mathrm{S}_{13}, \mathrm{~S}_{23}$ and $\mathrm{R}_{33}$ are attached on the moving platform.

For the first chain and second chain, The POC of end-effector is

$M_{b i}=\left[\begin{array}{l}t^{1}\left(\perp \mathrm{R}_{i 1}\right) \\ r^{1}\left(/ / \mathrm{R}_{i 1}\right)\end{array}\right] \cup\left[\begin{array}{c}t^{1}\left(/ / \mathrm{P}_{i 2}\right) \\ r^{0}\end{array}\right] \bigcup\left[\begin{array}{l}t^{0} \\ r^{3}\end{array}\right]=\left[\begin{array}{c}t^{2}\left(\perp \mathrm{R}_{i 1}\right) \\ r^{3}\end{array}\right],(i=1,2)$

For the third chain,

$M_{\mathrm{b} 3}=\left[\begin{array}{c}t^{1}\left(/ / P_{31}\right) \bigcup t^{1}\left(\perp R_{31}\right) \\ \mathrm{r}^{1}\left(/ / C_{31}\right)\end{array}\right] \cup\left[\begin{array}{c}\mathrm{t}^{1}\left(/ / \mathrm{p}_{32}\right) \\ \mathrm{r}^{0}\end{array}\right] \cup\left[\begin{array}{l}\mathrm{t}^{1} \perp\left(R_{31}\right) \\ \mathrm{r}^{1}\left(/ / R_{33}\right)\end{array}\right]=\left[\begin{array}{c}\mathrm{t}^{3} \\ \mathrm{r}^{1}\left(/ / C_{31}\right)\end{array}\right]$

where $M_{b i}$ denotes the POC equation of the $i$ th chain, $\mathrm{t}^{i}$ represent the branched-chain terminal have translational degrees of freedom and the number of DOF is $i(i=1,2,3) . \mathrm{r}^{j}$ stands for the branched-chain terminal have rotational freedom and the number of DOF is $j(j=1,2,3)$.

In order to calculate the DOF of the mechanism, a sub-PM is defined as a mechanism only consists of two kinematic chains of the original PM. For the first sub-PM composed of the first chain and second chain, its number of displacement equation $\xi_{L 1}$ is

$\xi_{L_{1}}=\operatorname{dim}\left\{\mathrm{M}_{\mathrm{b}_{1}} \cup \mathrm{M}_{\mathrm{b}_{2}}\right\}=\operatorname{dim}\left\{\left[\begin{array}{c}\mathrm{t}\left(\perp R_{11}\right) \\ \mathrm{r}^{3}\end{array}\right]\right\}=5$

According to DOF formula proposed by Yang and Sun [8], the DOF of the first sub-PM is

$F_{s u b}=\sum_{\mathrm{i}=1}^{\mathrm{m}} f_{\mathrm{i}}-\sum_{\mathrm{j}=1}^{1} \xi_{L_{\mathrm{j}}}=10-5=5$

where $F$ is the DOF of the mechanism, $f_{i}$ is the DOF of the $i$ th kinematic pair. So the POC equation of the first sub-PM is 
$M_{P \mathrm{a}}(1-2)=M_{\mathrm{b} 1 \cap} \mathrm{Mb}_{\mathrm{b} 2}=\left[\begin{array}{c}\mathrm{t}^{2}\left(\perp R_{11}\right) \\ \mathrm{r}^{3}\end{array}\right] \cap\left[\begin{array}{c}\mathrm{t}^{2}\left(\perp R_{21}\right) \\ \mathrm{r}^{3}\end{array}\right]=\left[\begin{array}{c}t^{2}\left(\perp R_{11}\right) \\ r^{1}\left(/ / R_{33}\right)\end{array}\right]$

Similarly, the number of displacement equation $\xi_{L 2}$ for another independent closed loop, which is composed of the first chain and the third chain, is

$\xi_{L_{2}}=\operatorname{dim} .\left\{M_{b 1} \cup \mathrm{Mb}_{\mathrm{b}}\right\}=\operatorname{dim} .\left\{\left[\begin{array}{l}\mathrm{t}^{3} \\ \mathrm{r}^{3}\end{array}\right]\right\}=6$

Complementing Eq. (3) with (6), we obtain

$F=\sum_{i=1}^{m} f_{i}-\sum_{j=1}^{2} \xi_{L_{j}}=14-(5+6)=3$

Finally, the output characteristics of moving platform can be described as follows

$M_{P a}=M_{P a(1-2)} \cap M_{b 3}=\left[\begin{array}{c}t^{2}\left(\perp R_{11}\right) \\ r^{3}\end{array}\right] \cap\left[\begin{array}{c}t^{3} \\ r^{1}\left(/ / R_{33}\right)\end{array}\right]=\left[\begin{array}{c}t^{2}\left(\perp R_{11}\right) \\ r^{1}\left(/ / R_{33}\right)\end{array}\right]$

Eqs. (7) and (8) show that the number of DOF of the mechanism is 3 and the moving platform moves along $x$-, $z$-axis and rotates around $x$-axis.

\section{Kinematics analysis}

\subsection{Position and orientation solutions}

As shown in Fig. 1, the coordinate system $O-x y z$ is fixed to the fixed base where the origin point $O$ is located on the midpoint of the side $A_{1} A_{2}, x$-axis is aligned with the side $A_{1} A_{2}, y$-axis is parallel to the axis of $\mathrm{R}_{11}, z$-axis is defined by the right-hand rule. The moving coordinate system $O^{\prime}-x^{\prime} y^{\prime} z^{\prime}$ is fixed to the moving platform, where the original point $O^{\prime}$ is located on the midpoint of the side $B_{1} B_{2}$. Three axes, $x^{\prime}-, y^{\prime}-, z^{\prime}$ - axes of the moving frame are parallel to the axes of the fixes frame. $O A_{1}=O A_{2}=$ a, $O^{\prime} B_{1}=O^{\prime} B_{2}=\mathrm{b}$; distance between the point $O$ to the axis of $\mathrm{C}_{31}$ is $\mathrm{d}_{2} ; O^{\prime} B_{3}=\mathrm{d}_{1}, \theta$ is the attitude angle of the moving plating around $x$-axis. $\mathbf{T}=\left[\begin{array}{lll}x & 0 & z\end{array}\right]^{\mathrm{T}}$ is the position coordinate of the original point $O^{\prime}$. Attitude matrix $\mathbf{R}$ of the moving platform can be derived as follows

$\mathbf{R}=\left[\begin{array}{ccc}1 & 0 & 0 \\ 0 & \cos \theta & -\sin \theta \\ 0 & \sin \theta & \cos \theta\end{array}\right]$

\subsection{Inverse positions solution}

The inverse positions solution of the PM is that finding the input line displacements $l_{1}, l_{2}, l_{3}$ when the coordinate $(x, 0, z)$ and attitude angle $\theta$ are given. The coordinates of $B_{1}$ and $B_{2}$ in the fixed coordinates can be computed easily, and $\mathbf{B}_{1}=\left[\begin{array}{lll}x-b & 0 z\end{array}\right]^{\mathrm{T}}, \mathbf{B}_{2}=\left[\begin{array}{lll}x+b & 0 z\end{array}\right]^{\mathrm{T}}$

According to Eq.(9), the coordinate of point $B_{3}$ is

$\mathbf{B}_{3}=\mathbf{R B}_{3 O^{\prime}}+\mathbf{T}$

where $B_{30}$ ' is the coordinate in the moving coordinate system

Substituting the known parameters into Eq. (10), we have

$\mathbf{B}_{3}=\left[\begin{array}{llll}x & r_{1} \cos \theta & r_{1} & \sin \theta+z\end{array}\right]^{\mathrm{T}}$

According to the constraint of the rod length, the position equation can be gotten.

$l_{1}^{2}=(e-x)^{2}+z^{2}$

$l_{2}^{2}=(e+x)^{2}+z^{2}$

$l_{3}^{2}=\left(r_{2}-d_{1} \cos \theta\right)^{2}+\left(z+d_{1} \sin \theta\right)^{2}$

where $e=a-b$. When three independent parameters $x, z, \theta$ are given, $l_{1}, l_{2}, l_{3}$ can be solved. It is obvious that the inverse position has only one solution. 


\subsection{Forward position solutions}

The forward position solutions is to define the coordinate $x, y, z$, of point $O^{\prime}$ in the moving frame when all input displacements of the three actuators, $l_{1}, l_{2}, l_{3}$ are given.

From Eq. (11), (12), (13), we obtain

$$
\begin{aligned}
& x=\frac{l_{2}^{2}-l_{1}^{2}}{4 e} \\
& z= \pm \sqrt{\frac{4 e l_{1}^{2}-4 e+l_{1}^{2}-l_{2}^{2}}{4 e}}
\end{aligned}
$$

$M \sin \theta+N \sin \theta=\mathrm{k}$

where $M=2 d_{1} d_{2}, N=-2 d_{1} d_{2}, K=\mathrm{z}^{2}+d_{2}^{2}+d_{1}^{2}-l_{3}^{2}$

From Eq. (16), we obtain

$\theta=2 \tan ^{-1} \frac{M \pm \sqrt{M^{2}+N^{2}-K^{2}}}{M+K}$

Since the structural limitation, sign " \pm " in (15) can only be selected sign "+" and sign "_" should be deleted. That is to say, $z$ just has one solution. Thus, when three input parameters $l_{1}, l_{2}, l_{3}$ are given, position parameters $x, z$ and pose angle $\theta$ can be solved. The forward position has two solutions.

From Eqs. (11), (12) and (13), the function relationship between the output and the input parameters can be described as $x=f\left(l_{1}, l_{2}\right), z=f\left(l_{1}, l_{2}\right), \theta=f\left(l_{1}, l_{2}, l_{3}\right)$. Thus this mechanism has weakly coupling property and is easy to complete real-time control.

\subsection{Velocity analysis}

Differentiating Eqs. (11)-(13) with respect to time, then obtain

$l_{1} \dot{l}_{1}=(e-x) \dot{x}+z \dot{z}$

$l_{2} \dot{l}_{2}=(e+x) \dot{x}+z \dot{z}$

$l_{3} \dot{l}_{3}=\left(z+d_{1} \sin \theta\right) \dot{z}+\left[\left(R-d_{1} \cos \theta\right) \sin \theta+\left(z+d_{1} \sin \theta\right) \cos \theta\right] d_{1} \dot{\theta}$

Rewriting them as matrix form, have

$\mathbf{J}_{d i r} \mathbf{v}=\mathbf{J}_{i n v} \dot{\boldsymbol{s}}$

where $\boldsymbol{v}=\left[\dot{l}_{1}, \dot{l}_{2}, \dot{l}_{3}\right]^{\mathrm{T}}$ represents the output velocity vector of the moving platform and $\dot{\boldsymbol{s}}=[\dot{x}, \dot{y}, \dot{\theta}]^{\mathrm{T}}$ represents the input velocity vector of the actuators. While $\mathbf{J}_{d i r}$ and $\mathbf{J}_{i n v}$ stand for the direct and the inverse Jacobian matrices, respectively, and

$$
\mathbf{J}_{d i r}=\left[\begin{array}{ccc}
l_{1} & 0 & 0 \\
0 & l_{2} & 0 \\
0 & 0 & l_{3}
\end{array}\right] \quad \mathbf{J}_{\text {inv }}=\left[\begin{array}{ccc}
e-x & z & 0 \\
e+x & z & 0 \\
0 & m & n+p
\end{array}\right]
$$

where $\left.m=z+d_{1} \sin \theta, n=\left(d_{2}-d_{1} \cos \theta\right)\right) d_{1} \sin \theta, p=\left(z+d_{1} \sin \theta\right) d_{2} \cos \theta$.

By combining Eq. (21) with Eq. (22), the formulate for solving the inverse/forward velocities are derived as follows

$$
\begin{aligned}
& {\left[\begin{array}{c}
\dot{x} \\
\dot{z} \\
\dot{\theta}
\end{array}\right]=\left[\begin{array}{ccc}
e-x & z & 0 \\
e+x & z & 0 \\
0 & m & n+p
\end{array}\right]^{-1}\left[\begin{array}{lll}
l_{1} & 0 & 0 \\
0 & l_{2} & 0 \\
0 & 0 & l_{3}
\end{array}\right]} \\
& {\left[\begin{array}{l}
\dot{l}_{1} \\
\dot{l}_{2} \\
\dot{l}_{3}
\end{array}\right]=\left[\begin{array}{lll}
l_{1} & 0 & 0 \\
0 & l_{2} & 0 \\
0 & 0 & l_{3}
\end{array}\right]^{-1}\left[\begin{array}{ccc}
e-x & z & 0 \\
e+x & z & 0 \\
0 & m & n+p
\end{array}\right]\left[\begin{array}{c}
\dot{x} \\
\dot{z} \\
\dot{\theta}
\end{array}\right]}
\end{aligned}
$$




\subsection{Forward acceleration}

According to the second order influence coefficient method $[9,10]$, the acceleration $a$ is derived as below

$\boldsymbol{a}=\dot{l}^{\mathrm{T}} \mathbf{H} \dot{l}+\ddot{\jmath}$

where " $\mathrm{a}$ " is the acceleration vector, it can derived as $\boldsymbol{a}=\left[a_{x}, a_{z}, \alpha_{x}\right]^{\mathrm{T}}$. H represent Hesse matrix, and

$\mathbf{H}=\left[\begin{array}{lll}\mathbf{H}_{11} & \mathbf{H}_{12} & \mathbf{H}_{13} \\ \mathbf{H}_{21} & \mathbf{H}_{22} & \mathbf{H}_{23} \\ \mathbf{H}_{31} & \mathbf{H}_{32} & \mathbf{H}_{33}\end{array}\right]$

$\mathbf{H}_{i j}=\frac{\partial^{2} u}{\partial l_{i} \partial l_{j}}=\left(\begin{array}{lll}\frac{\partial^{2} u_{1}}{\partial l_{i} \partial l_{j}} & \frac{\partial^{2} u_{2}}{\partial l_{i} \partial l_{j}} & \frac{\partial^{2} u_{3}}{\partial l_{i} \partial l_{j}}\end{array}\right)$

where $u_{1}=l_{1}^{2}-(e-x)^{2}+z^{2}, u_{2}=l_{2}^{2}+(x+e)^{2}+z^{2}, u_{3}=l_{3}^{2}+\left(d_{2}-d_{1} \cos \theta\right)^{2}+\left(z+d_{1} \sin \theta\right)^{2}$.

$\mathbf{J}=\mathbf{J}_{d i r}^{-1} \mathbf{J}_{i n v}=\left[\begin{array}{ccc}e-x & z & 0 \\ e+x & z & 0 \\ 0 & z+d_{1} \sin \theta & \left(d_{2}+z \cot \theta\right) d_{1} \sin \theta\end{array}\right]$

By substituting Eqs. (26), (27), (28) into Eq. (25), the forward acceleration solutions will be obtained as well.

\section{Kinematic simulation}

If all structural sized of the mechanism are given, and $a=100 \mathrm{~mm}, b=82.27 \mathrm{~mm}, d_{1}=95 \mathrm{~mm}$, $d_{2}=145 \mathrm{~mm}$, the virtual prototype model of the $2 \mathrm{~T} 1 \mathrm{R} \mathrm{PM}$ is built by PRO/E software in Fig. 2 . Then the model is imported to ADAMS software and the kinematics simulation can be realized easily. The prismatic pairs of the three chains are selected to the actuators, and their input functions are given, respectively, and $l_{1}=66.2+15 * \sin (100 \mathrm{~d} * \mathrm{t}), l_{2}=67.4+40 * \sin (140 \mathrm{~d} * \mathrm{t}), l_{3}=51.4+20 * \sin (120 \mathrm{~d} * \mathrm{t})$. Time range is from 0 to 5 second. The generalized displacement, velocity and acceleration curves of the mechanism are solved (see Figs. 3-7).

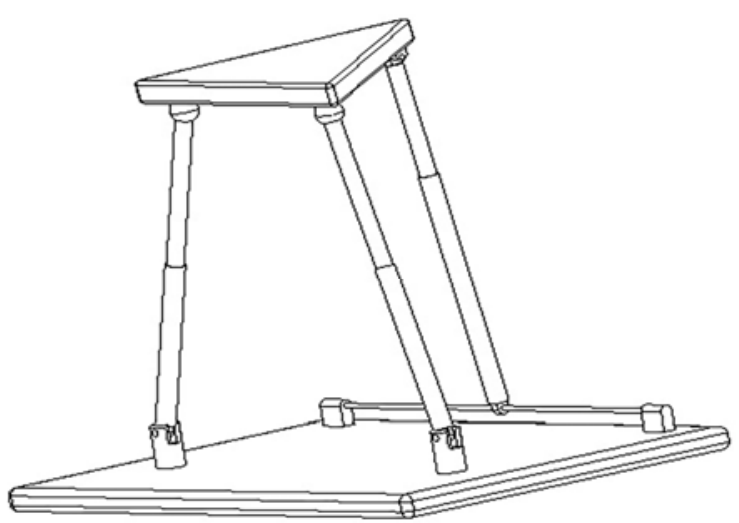

Fig. 2 The virtual prototype model of the 2T1R PM

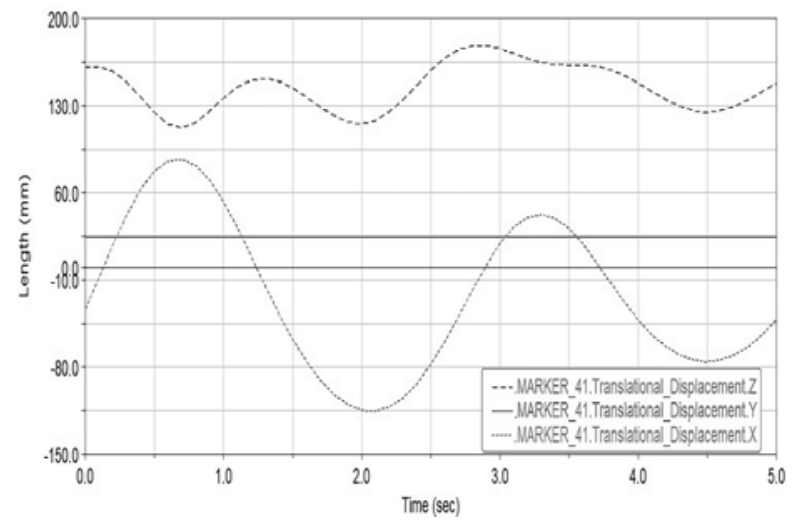

Fig. 3 displacement 


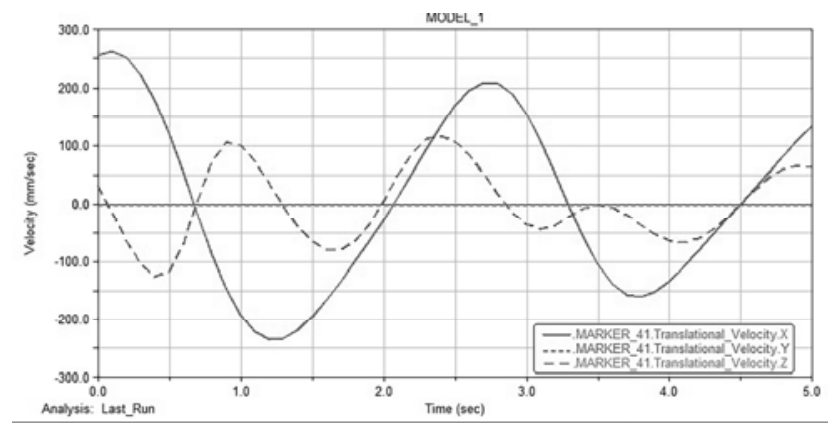

Fig. 4 velocity

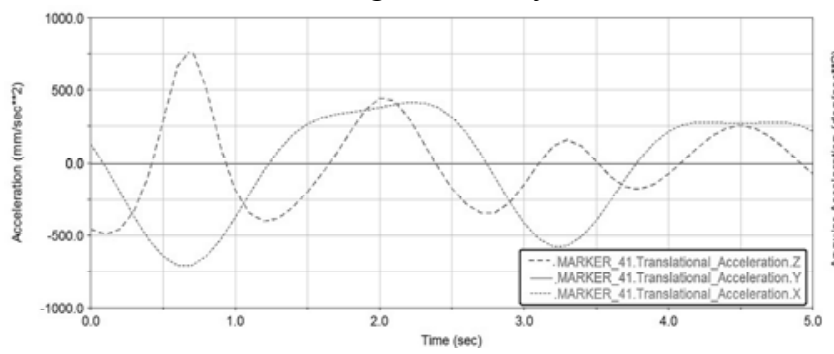

Fig. 6 acceleration

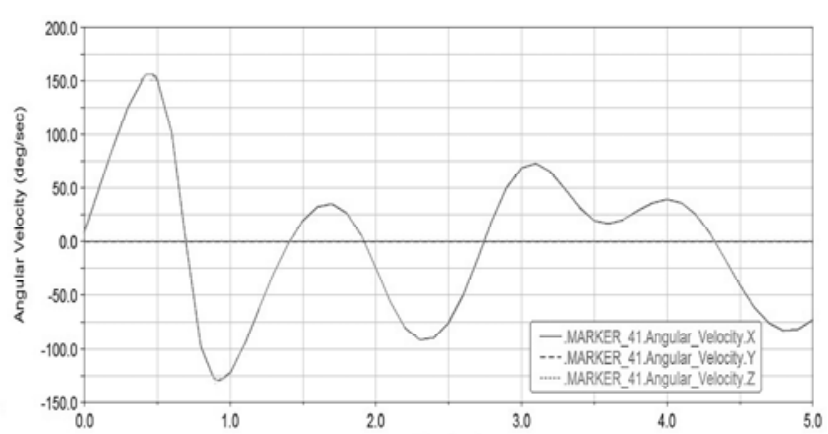

Fig. 5 angular velocity

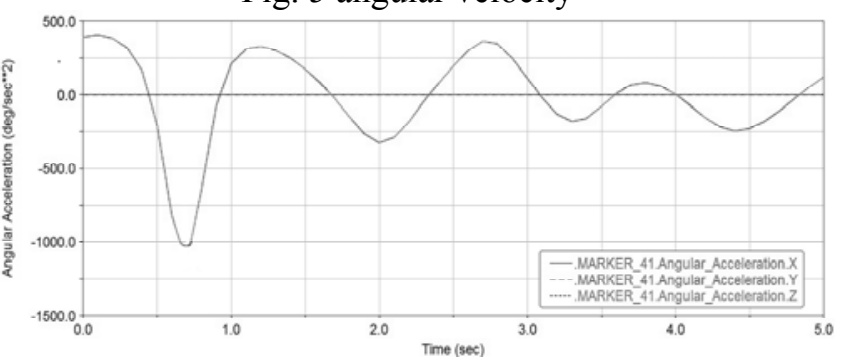

Fig. 7 angular acceleration

\section{Summary}

A new spatial parallel mechanism consisted of two RPS-type chains and one CPR-type chain. The moving platform has three DOFs i.e. two translation and one rotation. The number of the mobility is computed and the output motion characteristics of the parallel mechanism are analyzed based on the POC theory. The inverse and forward kinematic problems are discussed. According to the relationship between output and input, the robot has weak coupling property and is easy to complete real-time control. The virtual prototype is established based on PRO/E software and simulated by ADAMS software. The manipulator has widely potential applications in the fields of parallel machine tools, picking and packaging robots and micromanipulation robot.

\section{Acknowledgments}

Authors wish to acknowledge the supports by the National Natural Science Foundation of China under grant 50905055, Program for Innovative Research Team (in Science and Technology) in University of Henan Province under grant 15IRTSTHN008, and Program for Innovative Research Team of Henan University of Science and Technology under grant 2015XTD012.

\section{References}

[1] R. Calvel, DELTA, a fast robot with parallel geometry. International Symposium on Industrial Robotics. Switzerland, 1988, p. 91-100.

[2] C. Gosselin, J. Hamel, The agile eye: a high-performance three-degree-of-freedom cameraorienting device. International Conference on Robotics and Automation. San Diego, 1994, p. 781-786.

[3] K H Hunt. Structural kinematics of in-parallel-actuated robot-arms. Journal of Mechanical Design. Vol. 105 (1983) No. 4,p. 705-712

[4] S Refaat, J M Hervé, Nahavandi S, et al. Asymmetrical three-DOFs rotational-translational parallel-kinematics mechanisms based on Lie group theory. European Journal of Mechanics A/Solids. Vol. 20 (2006) No. 3,p. 550-558.

[5] Yanbin Zhang, Kwun-lon Ting, Design and Analysis of a Spatial 3-DOF Parallel Manipulator with 2T1R-Type. International Journal of Advanced Robotic Systems. Vol. 226 (2013) No. 10, p. 705-712. 
[6] Yanbin Zhang, Hongzhao Liu, Xin Wu. Kinematics analysis of a novel parallel manipulator. Mechanism and Machine Theory. Vol. 44 (2009) No. 9, p. 1648-1657..

[7] Tingli Yang, Theory and Application of Robot Mechanism Topology science press, 2012.(In Chinese).

[8] T L Yang, D J Sun. A general formula of degree of freedom for parallel mechanisms. International Design Engineering Technical Conferences and Computers and Information in Engineering Conference, American Society of Mechanical Engineers. New York, 2008, p. 1379-1390.

[9] H. B. Wang, Z. Huang. Dynamic force analysis of n.-df multiloop complex spatial mechanisms. Mechanism and Machine Theory. Vol. 27 (1990) No. 1, p. 167-173.

[10] $\mathrm{Lu} \mathrm{Y,} \mathrm{Hu} \mathrm{B.} \mathrm{Analyzing} \mathrm{kinematics} \mathrm{and} \mathrm{solving} \mathrm{active/constrained} \mathrm{forces} \mathrm{of} \mathrm{a} \mathrm{3SPU+UPR}$ parallel manipulator. Mechanism and Machine Theory. Vol. 42 (2007) No.10, p. 1298-1313. 